\title{
Pipelined Vedic-Array Multiplier Architecture
}

\author{
Vaijyanath Kunchigi \\ Jawaharlal Nehru Technological University Hyderabad, AP, INDIA \\ E-mail: vaijanath.k@gmail.com \\ Linganagouda Kulkarni \\ B. V. B. College of Engineering and Technology, Hubli, INDIA \\ E-mail: linganagouda@yahoo.co.uk \\ Subhash Kulkarni \\ ECE, PESIT, South Campus, Bangalore, INDIA \\ E-mail: subhashsk@gmail.com
}

\begin{abstract}
In this paper, pipelined Vedic-Array multiplier architecture is proposed. The most significant aspect of the proposed multiplier architecture method is that, the developed multiplier architecture is designed based on the Vedic and Array methods of multiplier architecture. The multiplier architecture is optimized in terms of multiplication and addition to achieve efficiency in terms of area, delay and power. This also gives chances for modular design where smaller block can be used to design the bigger one. So the design complexity gets reduced for inputs of larger number of bits and modularity gets increased. The proposed Vedic-Array multiplier is coded in Verilog, synthesized and simulated using EDA (Electronic Design Automation) tool XilinxISE12.3, Spartan 3E, Speed Grade-4. Finally the results are compared with array and booth multiplier architectures. Proposed multiplier is better in terms of delay and area as compared to booth multiplier and array multiplier respectively. The proposed multiplier architecture can be used for high-speed requirements.
\end{abstract}

Index Terms-Vedic, Array, Multiplier, Booth, High Speed.

\section{INTRODUCTION}

Multiplier is one of the key hardware blocks in designing arithmetic, signal and image processors. Many transform algorithms like the basic building blocks in Fast Fourier transforms, DCT, DFT etc., make use of multipliers. High performance multipliers [1] using Vedic mathematics [2] are proposed and conclude that it is suitable for high-speed complex arithmetic circuits [3]. The basic idea behind all these attempts was the fast implementation of the multiplier and addition [4] of the partial products [5]. Innumerable schemes have been proposed for realization of this operation [6]. With advances in technology, many researchers have tried to design multipliers using Vedic sutras [7], which offer high speed [8], low power consumption [9], and regularity of layout and less area or even combination of them in multiplier [10].
Multiplier is time-consuming operations in many of the digital signal processing applications [11] and computation can be reduced using the Vedic sutras and the overall processor [12] performance can be improved for many applications. Therefore, the goal is to create hybrid architectures that is comparable in speed, area and power, but requires less area than a design using a standard multiplier. The motivation behind this work is to explore the Design and implementation of hybrid multiplier architecture. The proposed pipelined VedicArray multiplier is based on the Vedic Sutras.

This paper is organized as follows. Section II gives the overview of Vedic mathematics, Section III briefs about proposed architecture section IV discusses about synthesis and simulation results analysis and section $\mathrm{V}$ about conclusion.

\section{VEDIC MATHEMATICS}

Vedic Mathematics (VM) is an ancient system of mathematics that was re-discovered by Sri Bharati Krishna Tirthaji between 1911 and 1918. Tirthaji (18841960) was an Indian scholar well versed in the areas of Sanskrit, English, Mathematics, Astronomy and many other areas of science. He deciphered ancient Indian texts, known as the "Ganita Sutras", (which means mathematics) to discover 16 short verses, known as "Sutras". These sutras, when applied correctly, will enable the user to solve many types of math problems mentally without having to use pencil and paper in a fraction of the time in would take otherwise. Tirthaji wrote sixteen books, one for each sutra, describing the application of each to the solution of math problems. Unfortunately, these books were lost. Tirthaji attempted to re-write all of these books from memory, but, was only able to complete the first volume entitled "Vedic Mathematics" before his death. This book, which is available today, is the seminal work on Vedic Mathematics.

Swami Bharati Krishna Tirtha (1884-1960), former Jagadguru Sankaracharya of Puri culled a set of 16 Sutras (aphorisms) and $13 \mathrm{Sub}$ - Sutras (corollaries) from the Atharva Veda. He developed methods and techniques for 
amplifying the principles contained in the aphorisms and their corollaries, and called it Vedic Mathematics. According to him, there has been considerable literature on Mathematics in the Veda-sakhas. Unfortunately most of it has been lost to humanity as of now. This is evident from the fact that while, by the time of Patanjali, about 25 centuries ago, 1131 Veda-sakhas were known to the Vedic scholars, only about ten Veda-sakhas are presently in the knowledge of the Vedic scholars in the country. The Sutras apply to and cover almost every branch of Mathematics. They apply even to complex problems involving a large number of mathematical operations. Application of the Sutras saves a lot of time and effort in solving the problems, compared to the formal methods presently in vogue. Though the solutions appear like magic, the application of the Sutras is perfectly logical and rational. The computation made on the computers follows, in a way, the principles underlying the Sutras. The Sutras provide not only methods of calculation, but also ways of thinking for their application.

\section{PROPOSED ARCHITECTURE}

The proposed 4 bit multiplier, gives an 8 bit product $(\mathrm{P}=\mathrm{A} * \mathrm{~B})$, where $\mathrm{A}$ and $\mathrm{B}$ shall be unsigned numbers. The multiplier is of type Vedic-Array multiplier with bit-pair evaluation. Instead of making one straightforward multiplication as $a_{3} a_{2} a_{1} a_{0} * b_{3} b_{2} b_{1} b_{0}=p_{7} p_{6} p_{5} p_{4} p_{3} p_{2}$ $\mathrm{p}_{1} \mathrm{p}_{0}$ this multiplier carries out the multiplication in two steps. First, four 2 bit multiplications carried out, creating four partial 4-bit products. The four 4-bit partial products are added together to create the final 8-bit product. Using multiplexers can do multiplication. With this method is the hardware cost kept low to implement the multiplier. Also the work to implement the multiplier can be kept low, since only three different types of non-complex blocks are needed to be used to build up the entire multiplier.

$$
\begin{aligned}
& \text { Ex: } \quad a=0110 \\
& \underline{b}=1010 \\
& \left.\begin{array}{rc}
\mathrm{x} & 0100 \\
\mathrm{y} & 0010 \\
\mathrm{z} & 0100 \\
\mathrm{w} & 0010
\end{array}\right\} \\
& \mathrm{Sa}^{5} \quad \mathrm{Sa}^{0} \\
& 011100 \\
& \mathrm{w}^{3} \quad \mathrm{w}^{0} \\
& 0010 \\
& 00111100
\end{aligned}
$$

Fig 1: How the multiplier perform the multiplication.

The overall structure of the multiplier is as shown fig2, where multiplication can be done using multiplexers, and the addition is carried out in two steps i.e., carry save addition and carry propagate addition for high speed. In this technique, intermediate results are always in a redundant form of two numbers. Two types of arrays have been proposed for the addition of the intermediate results. In the first type, the arrays are iterative with regular interconnection structure, permitting multiplication in time $O(n)$. The second type arrays are of tree form, permitting higher speed in $O(\log n)$ time, but the irregular form of a tree-array does not permit an efficient VLSI realization. The four different partial products are created with the four multiplexers. All four inputs of the multiplexers are four bits wide. The CPA and CSA adder consists of full adders and half adders. This optimization can be carried out as part of the synthesis operation. Both CSA-adders can be implemented identically at RTL level by moving the optimization to the synthesis operation.

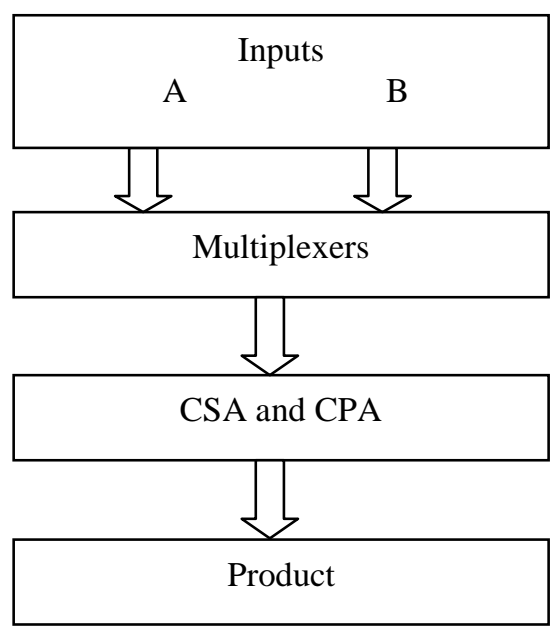

Fig 2. Block diagram of Vedic-Array Multiplier

\section{SYNTHESIS AND SIMULATION RESULT ANALYSIS}

Fig 2. shows the overall components involved in the design of 4-bit Vedic-Array architecture and it is implemented in Verilog HDL. Logic synthesis and simulation are done in Xilinx ISE simulator.

The synthesis results are compared with array and booth multiplier architecture. The results are shown in Table 1. The Table shows the area, speed and power estimation for Vedic-Array, Booth and Array multiplier architecture.

Simulation waveform, RTL Schematic and Technology Schematic of Vedic-Array, Booth and Array Multiplication Techniques is shown in below figures i.e., fig 3 to fig 11 .

Table 1: Area, Speed \& Power comparison of multipliers

\begin{tabular}{|c|c|c|c|}
\hline \multirow{2}{*}{ 4-bit } & \multicolumn{3}{|c|}{ Area, Speed and Power } \\
\cline { 2 - 4 } & $\begin{array}{c}\text { AREA } \\
(\text { SLICES })\end{array}$ & Speed $(n s)$ & $\begin{array}{c}\text { Power } \\
(\mathrm{mW})\end{array}$ \\
\hline $\begin{array}{c}\text { Vedic- } \\
\text { Array }\end{array}$ & 28 & 3.073 & 57.81 \\
\hline Array & 18 & 16.377 & 86.76 \\
\hline Booth & 32 & 6.307 & 53.00 \\
\hline
\end{tabular}




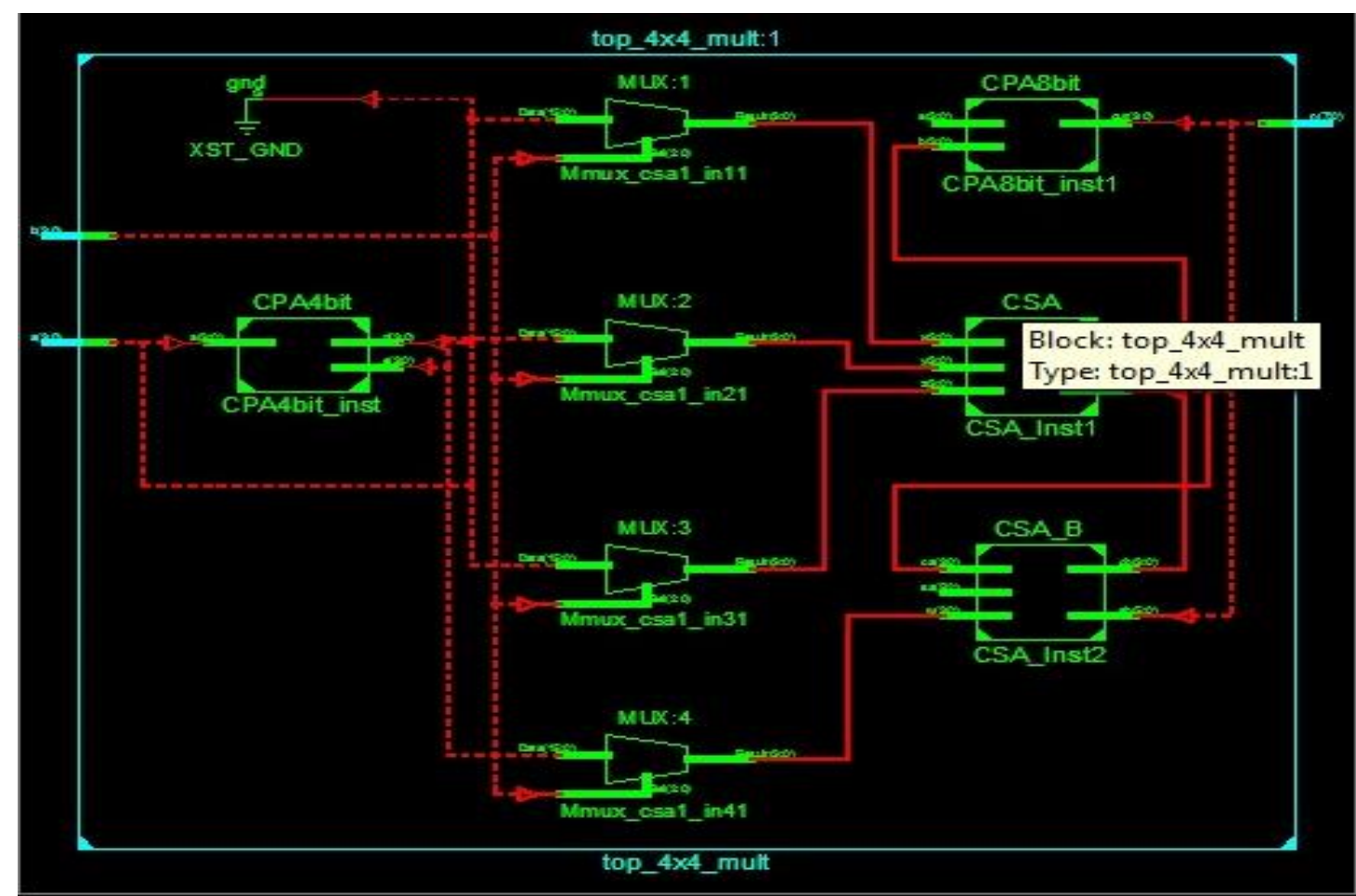

Fig 3: 4-bit Vedic-Array RTL schematic

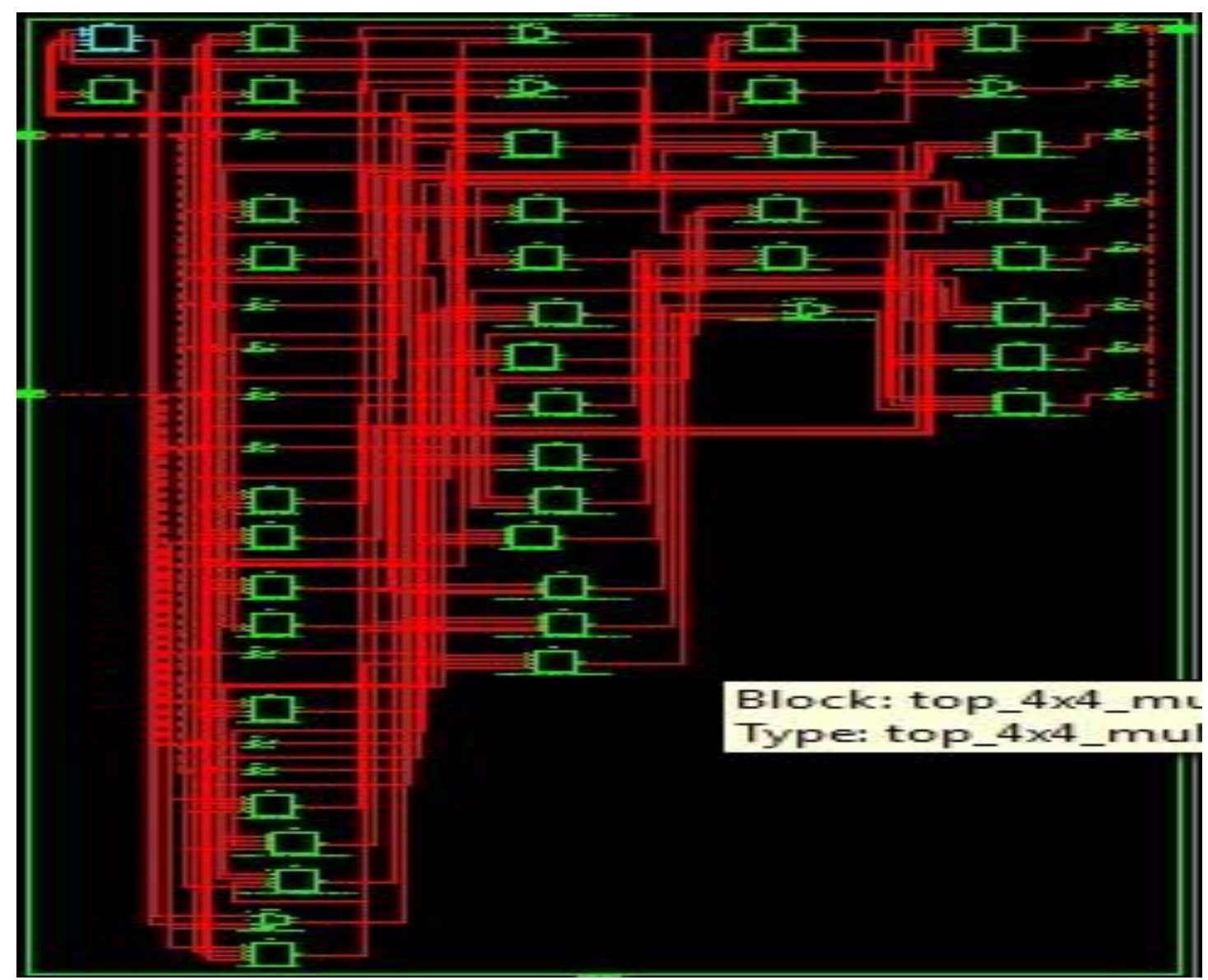

Fig 4: 4-bit Vedic-Array Technology Schematic 

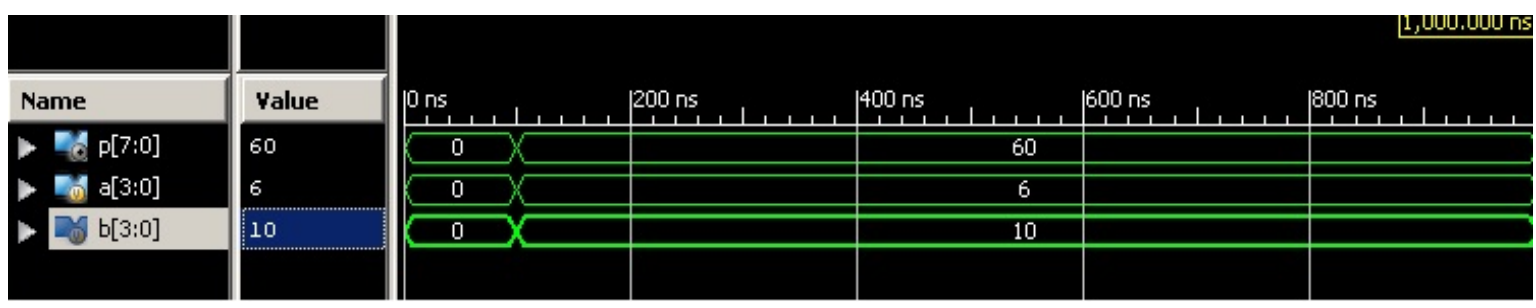

Fig 5: 4-bit Vedic-Array Simulation waveform

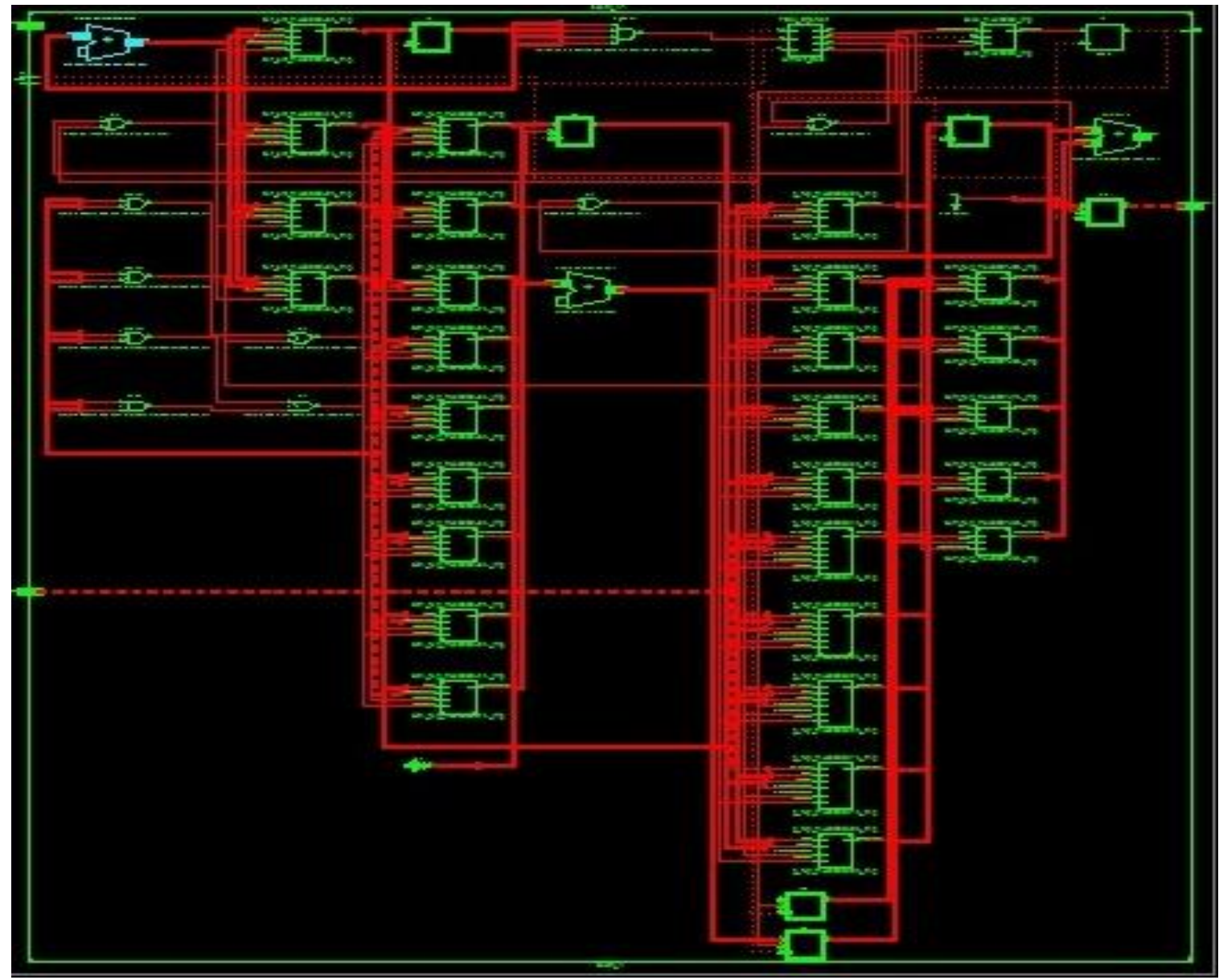

Fig 6: 4-bit Booth RTL

\begin{tabular}{|c|c|}
\hline Name & Value \\
\hline 16. done & 0 \\
\hline - product $7: 0]$ & 00001010 \\
\hline $16 \mathrm{clk}$ & 0 \\
\hline 18 enable & 1 \\
\hline - multiplier[3:0] & 1110 \\
\hline - 0 multiplicand[3; & 1011 \\
\hline
\end{tabular}

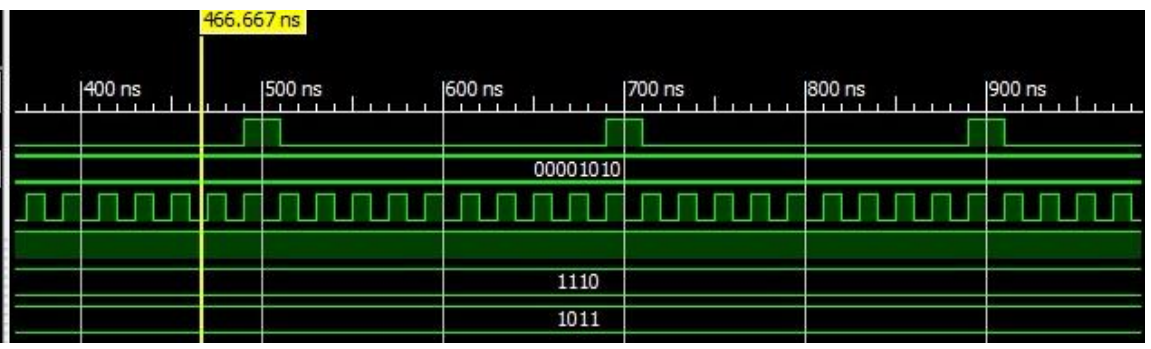

Fig 7: 4-bit Booth Simulation waveform 


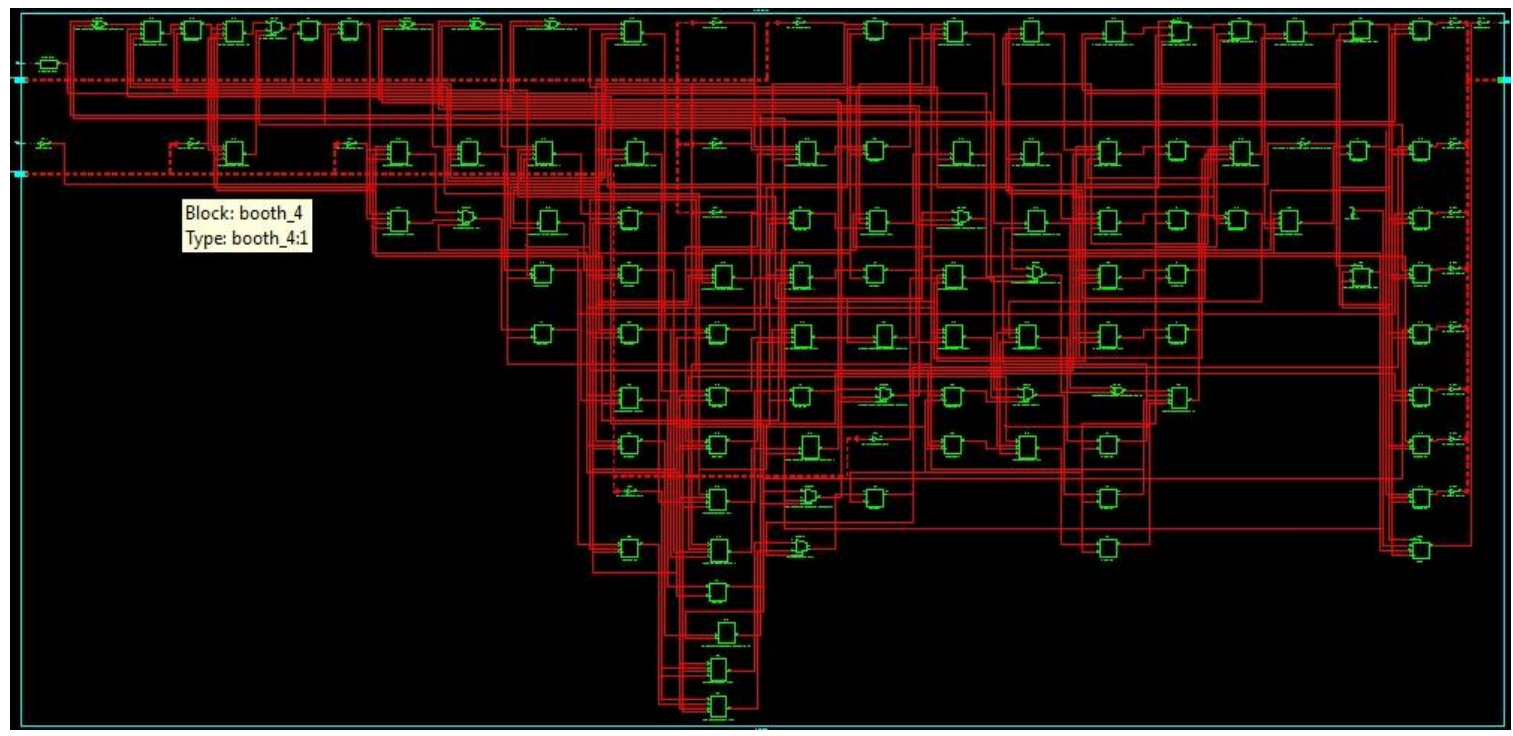

Fig 8: 4-bit Booth Tech Schematic

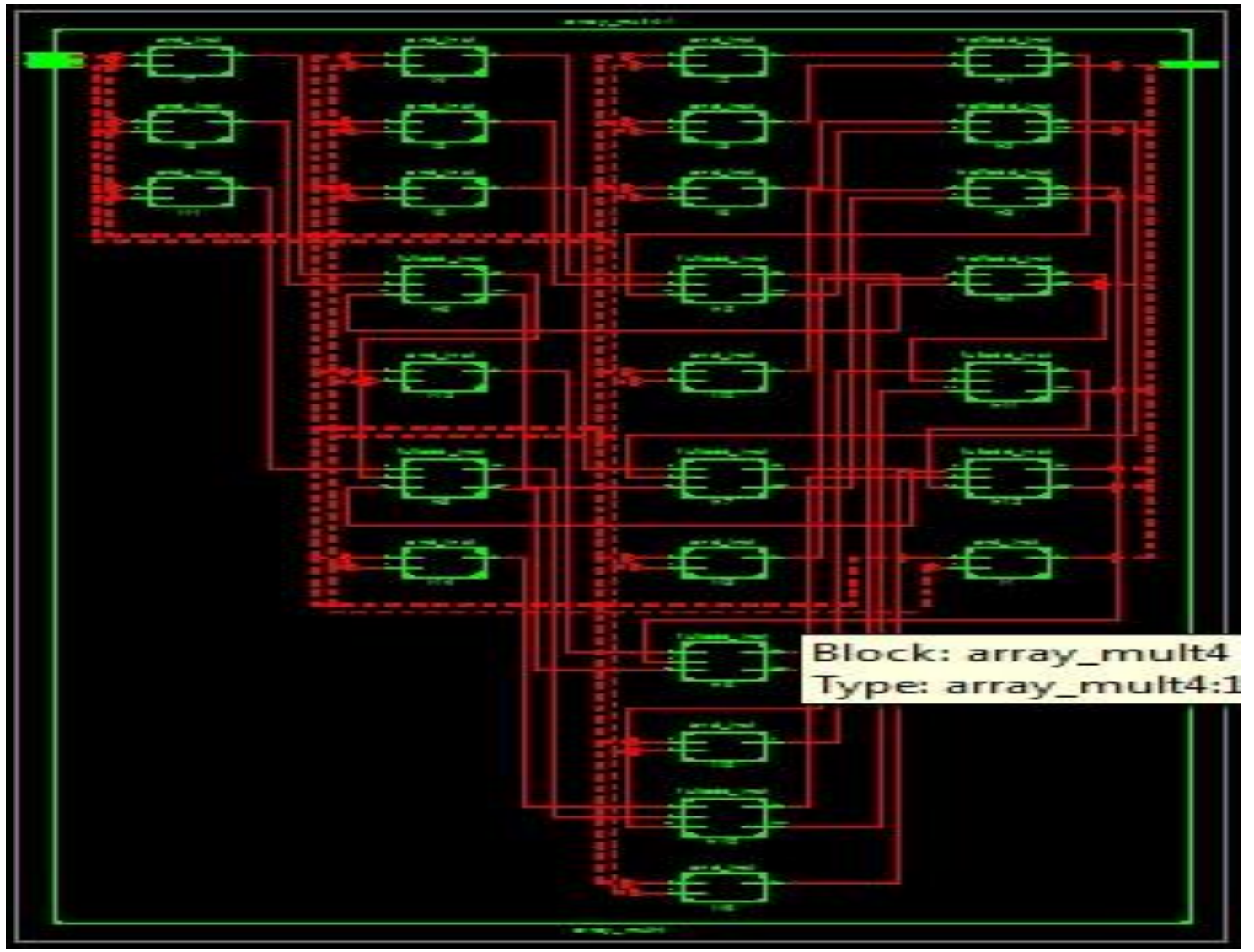

Fig 9: 4-bit Array RTL Schematic

\begin{tabular}{|l||l|}
\hline & \\
\hline Name & Value \\
\hline $\mathrm{a}[7: 0]$ & 21 \\
\hline $\mathrm{a}[\mathrm{B}: 0]$ & 3 \\
\hline $\mathrm{b}[3: 0]$ & 7 \\
\hline
\end{tabular}

$1,000.000 \mathrm{~ns}$

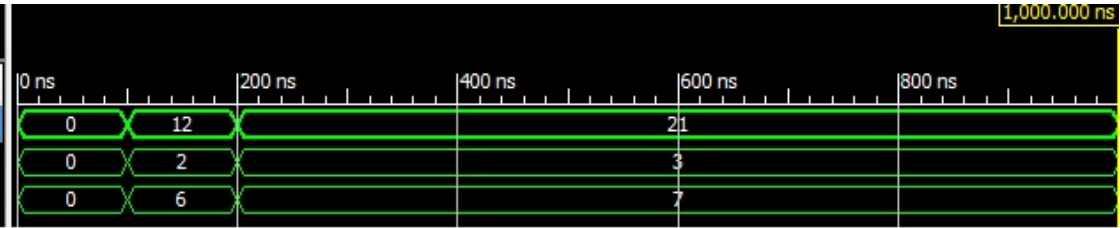

Fig 10: 4-bit Array Simulation Waveform 


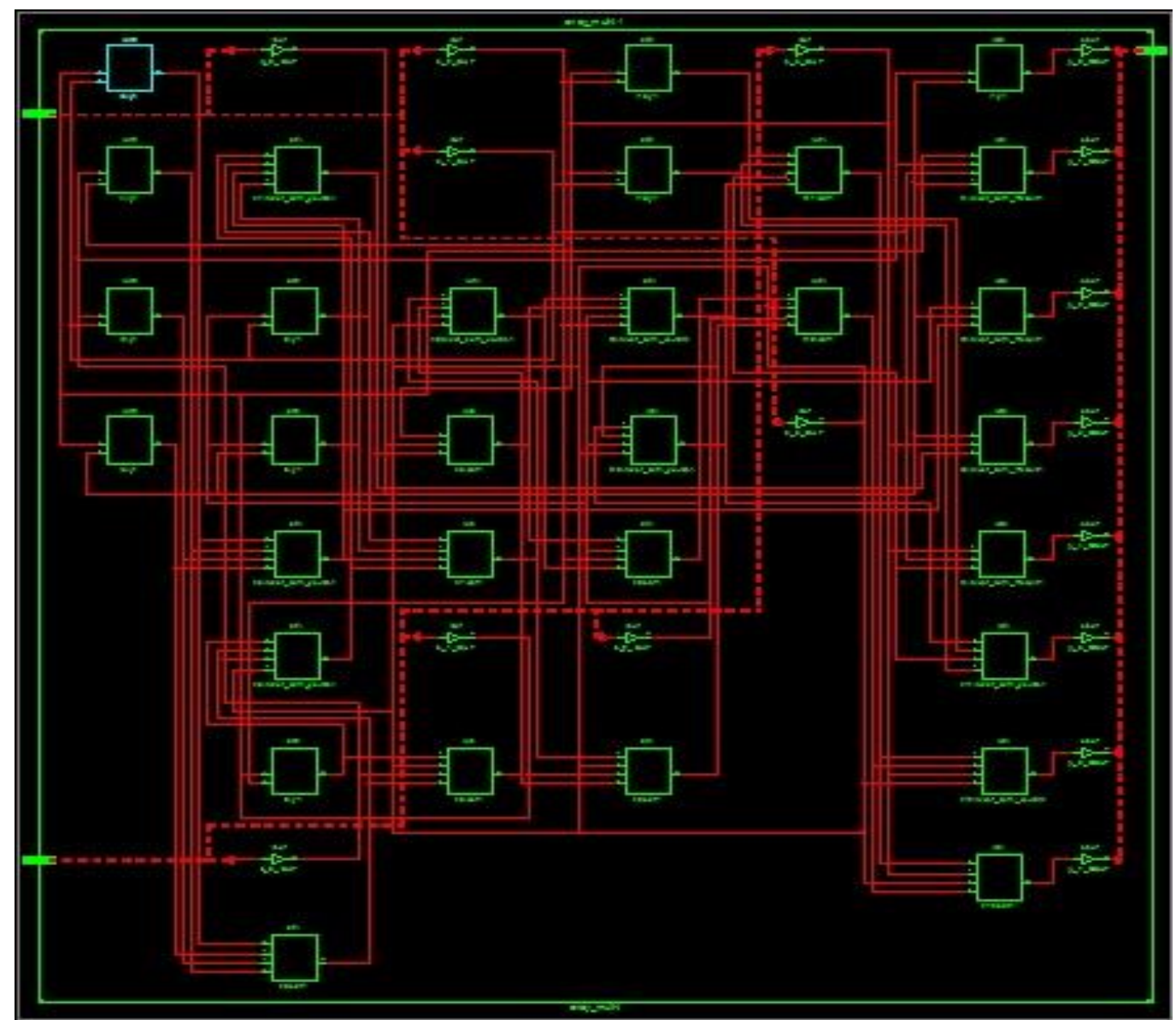

Fig 11: 4-bit RTL Technology Schematic

\section{CONCLUSION}

The structure of this Vedic-Array maintains the same level of regularity and results show significant improvement in delay. Pipelining approach reduces the critical path and useless signal transitions that are propagated through the array. Due to its regular and parallel structure it can be concluded that Vedic-Array multiplier architecture is efficient in terms of delay and Booth multiplier is superior with respect to complexity and power consumption. However Array multiplier requires more power consumption and gives optimum number of components required, but delay for this multiplier is larger than Vedic-Array and Booth Multiplier. Hence for high-speed requirements the VedicArray multiplier architecture can be applied, for low power requirement Booth's multiplier is suggested. Due to its structure, it suffers from a high carry propagation delay in case of multiplication of large number. Further the work can be extended for optimization of said multiplier to improve the power or to minimize the area. The idea of proposed hybrid multiplier architecture here may set path for future research in this direction. Future scope of research is to reduce power and area requirements.

\section{REFERENCES}

[1] Zhijun Huang, Miloš D. Ercegovac, "High-Performance Left-to-Right Array Multiplier Design," arith, pp.4, 16th IEEE Symposium on Computer Arithmetic (ARITH-16 '03), 2003.

[2] Ramalatha, M Dayalan, K D Dharani, P Priya, and S Deborah, "High speed energy efficient ALU design using Vedic multiplication techniques", ICACTEA, 2009. pp. 600-3, Jul 15-17, 2009.

[3] Shripad Kulkarni, "Discrete Fourier Transform (DFT) by using Vedic Mathematics"Papers on implementation of DSP algorithms/VLSI structures using Vedic Mathematics, 2006, www.edaindia.com, IC Design portal.

[4] M.B. Damle, Dr. S. S. Limaye, "Low-power Full Adder array-based Multiplier with Domino Logic," IOSR Journal of Electronics and Communication Engineering (IOSRJECE), ISSN : 2278-2834 Volume 1, Issue 1 (MayJune 2012), PP 18-22.

[5] Sumit R. Vaidya, D. R. Dandekar, "Performance Comparison of Multipliers for Power-Speed Trade-off in VLSI Design," RECENT ADVANCES in NETWORKING, VLSI and SIGNAL PROCESSING, ISSN: 1790-5117, ISBN: 978-960-474-162-5.

[6] Pushpalata Verma, K. K. Mehta, "Implementation of an Efficient Multiplier based on Vedic Mathematics Using EDA Tool," International Journal of Engineering and Advanced Technology (IJEAT), ISSN: 2249 -8958, Volume-1, Issue-5, June 2012. 
[7] Manoranjan Pradhan, Rutuparna Panda, Sushanta Kumar Sahu, "Speed Comparison of 16x16 Vedic Multipliers," International Journal of Computer Applications (0975 8887), Volume 21- No.6, May 2011.

[8] G.Ganesh Kumar, V.Charishma, "Design of High Speed Vedic Multiplier using Vedic Mathematics Techniques", International Journal of Scientific and Research Publications, Volume 2, Issue 3, March 20121 ISSN 22503153.

[9] Sumit Vaidya, Deepak Dandekar, "DELAY-POWER PERFORMANCE COMPARISON OF MULTIPLIERS IN VLSI CIRCUIT DESIGN", International Journal of Computer Networks \& Communications (IJCNC), Vol.2, No.4, July 2010.

[10] Sree Nivas A, Kayalvizhi N, “ Implementation of Power Efficient Vedic Multiplier", International Journal of Computer Applications (0975 - 8887) Volume 43- No.16, April 2012.

[11] Soma BhanuTej, "Vedic Algorithms to develop green chips for future", Volume 2, Issue ICAEM12, February 2012, ISSN Online: 22772677 ,ICAEM12,Jan20,2012,Hyderabad,India.

[12] Krishnaveni D., Umarani T.G., “ VLSI IMPLEMENTATION OF VEDIC MULTIP-LIER WITH REDUCED DELAY", International Journal of Advanced Technology \& Engineering Research (IJATER) National Conference on Emerging Trends in Technology (NCETTech), ISSN No: 2250-3536 Volume 2, Issue 4, July 2012.

[13] Ramachandran.S, Kirti.S.Pande, “ Design, Implementation and Performance Analysis of an Integrated Vedic Multiplier Architecture", International Journal Of Computational Engineering Research / ISSN: 2250-3005.
Vaijyanath Kunchigi, is Software Engineer working in multinational company. $\mathrm{He}$ is currently working toward the Ph.D degree in the Electronics and Communication in the JNTU University. His research interests include computer architecture, digital image processing and Software Development.

Linganagouda Kulkarni is Professor, Department of Electronics and Communication, B. V. B. College of Engineering and Technology, Hubli, India. He received his Ph.D. in image processing in 1997 from Mysore University India. His research areas include image processing, pattern recognition, and VLSI implementation of DIP algorithms.

Subhash Kulkarni, is Professor and Head. Department of Electronics and Communication, PESIT, south Campus, Bangalore. He received BE from Gulbarga University, M.Tech from IISc Bangalore and P.hD from IIT Kharagpur, India. He is Fellow of IETE and Fellow of Institution of Engineers. He has published more than 70 papers in reputed international and national journals. He is highly sought after as commendable resource person in Signal and Image Processing. His research interests are mathematical models in signal and image processing and computational architectures based on Vedic math's. He is an inspiration to the researchers in this area.

How to cite this paper: Vaijyanath Kunchigik, Linganagouda Kulkarni, Subhash Kulkarni,"Pipelined Vedic-Array Multiplier Architecture", IJIGSP, vol.6, no.6, pp. 58-64, 2014.DOI: 10.5815/ijigsp.2014.06.08 